\title{
Coupling Effects of Depletion Interactions in Colloidal Suspensions
}

\author{
Haixia Gao ${ }^{1}$, Zeshun Chen ${ }^{1}$, Chunshu $\mathrm{Li}^{1}$, Changming Xiao ${ }^{2 \#}$ \\ ${ }^{1}$ Department of Electronic Engineering, Hunan University of Science and Engineering, Yongzhou, China \\ ${ }^{2}$ Department of Physics, Hunan Normal University, Changsha, China \\ Email: "cmxiaocn@yahoo.com.cn
}

Received June 18, 2012; revised July 23, 2012; accepted July 31, 2012

\begin{abstract}
With the aid of Monte Carlo simulations and acceptance ratio method, the depletion interactions of the three-sphere system are studied in this paper, and the numerical results show that the depletion interactions in the three-sphere system are larger than that of the corresponding two-sphere system, i.e., the depletion interaction of the three-sphere system are coupled with each other. Furthermore, we find that the mechanisms of the coupling effect and the effect on depletion force from the geometry factor are the same. In addition, the numerical results also show that this coupling effect will be affected by both the volume fraction and separation of three-sphere system.
\end{abstract}

Keywords: Three-Sphere System; Coupling Effect of Depletion Interactions

\section{Introduction}

It's well known that the depletion interaction arises when two large spheres are immersed in the sea of small spheres. The mechanism about the depletion force was first described by Asakura and Oosawa (AO) with the concept of excluded volume [1]. According to AO, the depletion force is only attractive. However, both the experimental and simulation results show that besides the attractive part, there is also a repulsive part in the depletion interaction. So the mechanism for depletion interactions is not as simple as AO model. In recent years, many investigations on depletion interactions were carried out, and our understanding about them had been significantly improved [2-13]. It is known that the depletion force between two large-spheres will be strengthened if they are confined between two parallel plates [3], and the three-body depletion interactions in the equilateral triplets where the three large spheres are dispersed in equilateral-triangular configurations were studied, and the results show that the three-body interaction is attractive when the distance between them is small [4-7]. So the depletion interaction is not the additive one. It was also found that the depletion interactions can couple with each other when one sphere is suffered multi depletion forces at the same time $[10,11]$. In fact, the coupling effect is significant to the colloids, because the dynamic behavior of the particles and the structure of the system

\footnotetext{
*Supported by the Science Foundation of Hunan Educational Committee.

${ }^{\#}$ Corresponding author.
}

will be affected by this coupling effect. In this paper, we will study the depletion interactions among the three large spheres which are schematically illustrated by Figure 1(a). In this system, the large sphere B is placed between the two spheres A and C. Apparently B is suffered by two opposite depletion forces from $\mathrm{A}$ and $\mathrm{C}$ at the same time. However, we will find that, in the three-sphere system, the depletion forces acted on $\mathrm{B}$ are larger than that of the system without $C$. Furthermore, this three-sphere system is different to the equilateral triplets mentioned in Refs [4-7], and the results obtained from this system provide information not only about the three-body interaction but also about the coupling effect of depletion interactions. Maybe there are some relationships between the couple effect and geometrical confinement effect. In order to expose the character of the three-sphere system, a comparison with the corresponding two-sphere system demonstrated by Figure 1(b) is needed. So in this paper, the model and theory are presented in the second section, and the numerical results and a simple discussion will be given in the third section, finally a summary of our study is given in the last section.

\section{Theory and Model}

It is well know that a hard spheres mixture is characterized by the pair potential of

$$
u(d)=\left\{\begin{array}{cc}
\infty & d<d_{i j}=\left(\sigma_{i}+\sigma_{j}\right) / 2 \\
0 & d>d_{i j}
\end{array}\right.
$$


where $d$ is the distance between the two spheres in diameters $\sigma_{i}$ and $\sigma_{j}$, respectively. The force exerted on the big sphere of radius $R$ by a small sphere of radius $r$ can then be written as $k_{B} T \delta\left(d-d_{i j}\right)$. Consequently, the depletion force is the total force acted on the large sphere from the small spheres' of radius $r$, and is usually determined through the acceptance ratio method (ARM).

For ARM, if the potential and partition function of two systems are $V_{0}, Q_{0}$ and $V_{1}, Q_{1}$ respectively, where $V_{0}$ and $V_{1}$ are the external potentials corresponding to the two large spheres located at different positions, the free energy difference between these two systems is given by the following expression $[3,8,14]$,

$$
\begin{aligned}
\beta \Delta F & =-\ln \frac{Q_{1}}{Q_{0}}=-\ln \frac{\left\langle f\left[\beta\left(V_{1}-V_{0}\right)+C\right]\right\rangle_{0}}{\left\langle f\left[-\beta\left(V_{1}-V_{0}\right)-C\right]\right\rangle_{1}} \\
& =-\ln \frac{N_{10}}{N_{01}}
\end{aligned}
$$

where $N_{10}$ is the number of samples drawn out from $N$ simulated samples, which generated with the potential $V_{0}$ where $V_{1}$ is not infinite; $N_{01}$ is the number of samples drawn out from $N$ simulated samples, which generated with potential $V_{1}$ where $V_{0}$ is not infinite, and $f(x)=[1+\exp (x)]^{-1}$ is the Fermi function, and $C$ is a constant which is usually set to a value of 0 for a hard sphere system. Since the change of potential is only relates to the number of samples drawn out from the $N$ simulated samples, therefore the depletion interaction can be got through the ARM conveniently.

In this paper, we consider the system composed of three large spheres schematically described by Figure 1(a). In this system, the large sphere $B$ is suffered two depletion forces from $\mathrm{A}$ and $\mathrm{C}$ in opposite directions $f_{\mathrm{AB}}^{*}$, $f_{\mathrm{CB}}^{*}$ respectively, so the resultant force is $f=f_{\mathrm{AB}}^{*}-f_{\mathrm{CB}}^{*}$. For the sake of simplicity, the positions of spheres $A$ and $\mathrm{C}$ are fixed when $\mathrm{B}$ moves from the contact of $\mathrm{A}$ to the middle point of A and C. $h$ and $H$ are the separations of A and $\mathrm{B}, \mathrm{A}$ and $\mathrm{C}$, respectively. In order to expose the coupling effects, a comparison of the depletion interactions $f$ to that of the two-sphere system $f_{\mathrm{AB}}$ described by Figure 1(b) is needed. So we are going to determine the depletion interactions in both the two- and three-sphere systems respectively.

\section{Results and Discussions}

In the simulations, we consider the depletion forces of the hard spheres systems with a cell box of size $L_{x} \times$ $L_{y} \times L_{z}$, in which the three large sphere are placed along $z$ direction. Obviously, the small spheres are randomly distributed around the macro spheres to form a fluid. The size ratio of the macro- to micro-sphere $R / r$ is 5 , and the number of the micro-ions $N$ is determined by the

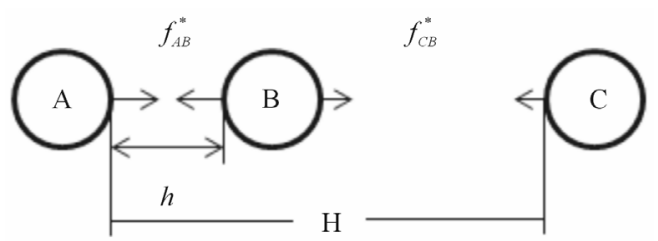

(a)

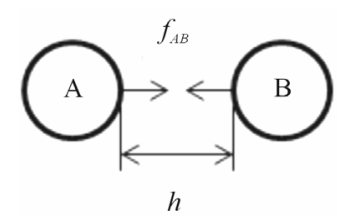

(b)

Figure 1. (a) Schematic diagram of the depletion interactions among the three large spheres A, B and C; (b) The depletion interaction between the two spheres $A$ and $B$. In (a) or (b), the positions of spheres $A$ and $C$ are fixed when $B$ moves from the contact of $A$ to the middle of $A$ and $C$. $h$ is the separation of spheres $A$ and $B$, and $H$ the separation of $A$ and $C$.

given volume fraction $\eta$, defined as $\eta=N V_{c} /\left(V-3 V_{m}\right)$ or $\eta=N V_{c} /\left(V-2 V_{m}\right)$ for the system of three- or twosphere respectively, where $V=L_{x} \times L_{y} \times L_{z}$ is the total volume of the cell box, $V_{c}=\frac{4}{3} \pi r^{s}$ is for the volume of the micro-sphere, $V_{m}=\frac{4}{3} \pi R^{3}$ denotes the volume of the macro-sphere. As the systems under consideration are not confined by geometry factors, the period boundary condition is applied to all the three directions of $X, Y$ and $Z$ in the Monte Carlo simulations. In addition, the configurations of the micro-spheres are sampled according to the Metropolis algorithm with the two macro-spheres A and $\mathrm{C}$ fixed while $\mathrm{B}$ moves from the contact of $\mathrm{A}$ to the middle point of $\mathrm{A}$ and $\mathrm{C}$. Each micro-sphere is orderly chosen involving a trial displacement. Except for an overlapping with the macro-spheres and the other micro-spheres, the new position of the micro sphere is randomly accepted. In our simulations, $1.0 \times 10^{5}$ Monte Carlo steps (MCS) are used for the equilibrium of the system and other $3.0 \times 10^{5} \mathrm{MCS}$ to collect data. In addition, the depletion potential is set as 0 while the two macro-spheres $\mathrm{A}$ and $\mathrm{B}$ are at contact, i.e., $h=0$. In this way, the depletion potentials, then the depletion forces in both the two- and three-sphere systems, $f_{\mathrm{AB}}, f$, are determined by ARM, and the results of the two system corresponding to the volume fractions $\eta=0.116,0.229$ and 0.341 are shown in Figures 2(a) and (b), Figures 3(a) and (b), Figures 4(a) and (b), respectively. The separation of sphere A and $\mathrm{C}$ of above systems is $H=18 r$. In addition, the depletion potential $F$ in unit of $k_{\mathrm{B}} T$ is plotted as a function of $h$, which is measured in unit of $2 r$, and the unit of depletion force is $\pi R \rho k_{\mathrm{B}} T$, where $\rho$ is 


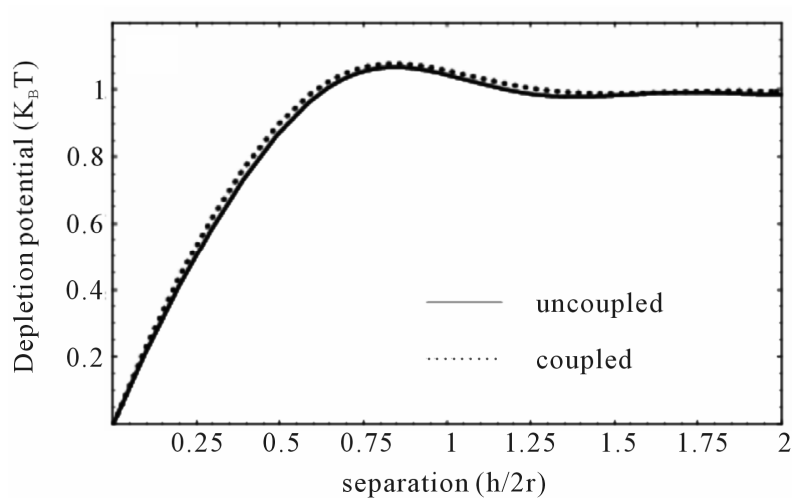

(a)

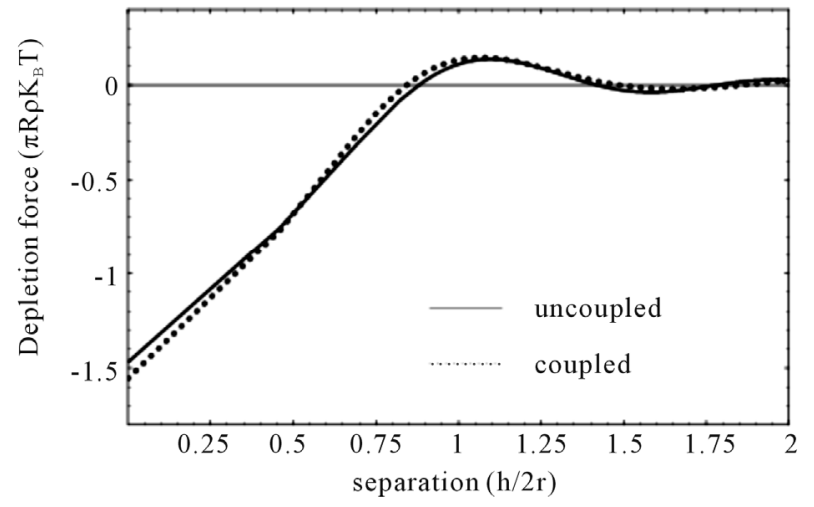

(b)

Figure 2. The depletion potentials (a) and the corresponding depletion forces (b) of the three-sphere system (described by the dashed line) and of the two-sphere system (by the solid line). The volume fraction is $\eta=\mathbf{0 . 1 1 6}$.

the number density of the small sphere. In Figures 2-4, the solid lines describe the depletion potentials or depletion forces of the systems composed of two-sphere, the dash-lines for that of the three-sphere systems'. From Figures 2-4, it's evident that, no matter the volume fraction is large or small, the depletion forces of the threesphere systems are larger than that of the corresponding two-sphere system. This result is not in accordance with the common sense of physics related to forces, therefore it is very interesting. As is known that, in the view of physics, when one object is suffered two forces in opposite directions at the same time, the resultant force will be smaller than the larger component one. However, the case considered here is that the sphere $\mathrm{B}$ is acted by the two opposite depletion forces from $\mathrm{A}$ and $\mathrm{C}$ at the same time, but the total force described by the dashed lines in Figures 2(b), 3(b) and 4(b) is larger than the larger component force from sphere A. So the additivity of depletion interactions is proved to be not true, at least for the case considered in this paper. Furthermore, it is reasonable to think that the two depletion forces impressed on the sphere B may couple with each other, and finally result in a strengthened total depletion force. This is the

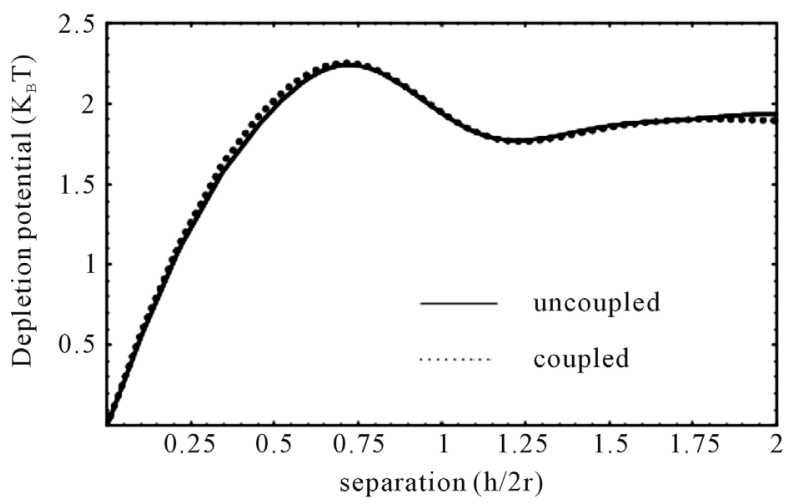

(a)

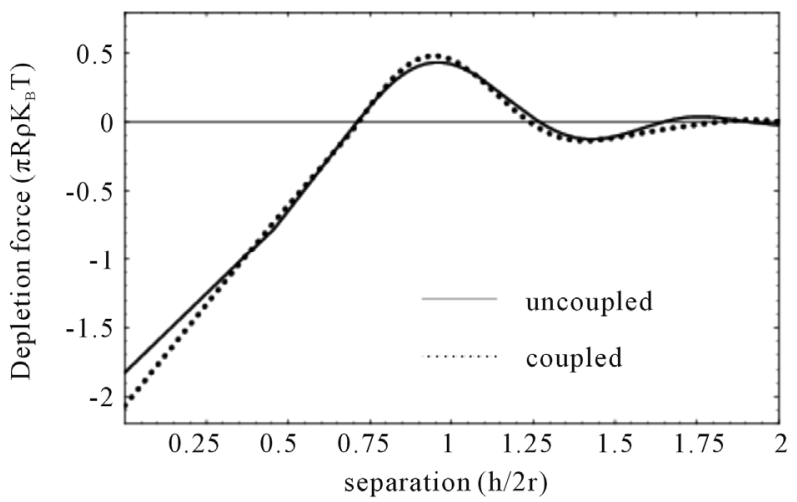

(b)

Figure 3. The depletion potentials (a) and the corresponding depletion forces (b) of the three-sphere system (described by the dashed line) and of the two-sphere system (by the solid line). The volume fraction is $\boldsymbol{\eta}=\mathbf{0 . 2 2 9}$.

coupling effect of the depletion interactions. Unfortunately the mechanism for the coupling effect is still unknown. However, we believe that there are some relations between the coupling effect and the effect on depletion force from the geometry factor. In fact, as is known that the depletion force between two spheres will be strengthened if they are confined by geometry factors, such as by two plates [3], or by cylinder [12], or by other spheres [13], etc. According to the geometry confinement, the large sphere $\mathrm{C}$ can be taken as a geometry factor of the system consisting of the two large spheres A and B, in this case, the depletion force between A and B must be strengthened due to the presence of large sphere C. So the intrinsic of the two kind of effect on depletion interactions are the same. On the hand, based on the fact that there also depletion forces between the geometry factors and the large spheres, the effect of geometry confinement can ever be taken as a special case of the coupling effect.

On the other hand, from Figures 2(b) and 4(b) we also find that, in Figure 2(b), the maximum value of the depletion force of the three-sphere system is near to -1.55 , but the value of the corresponding two-sphere system is 


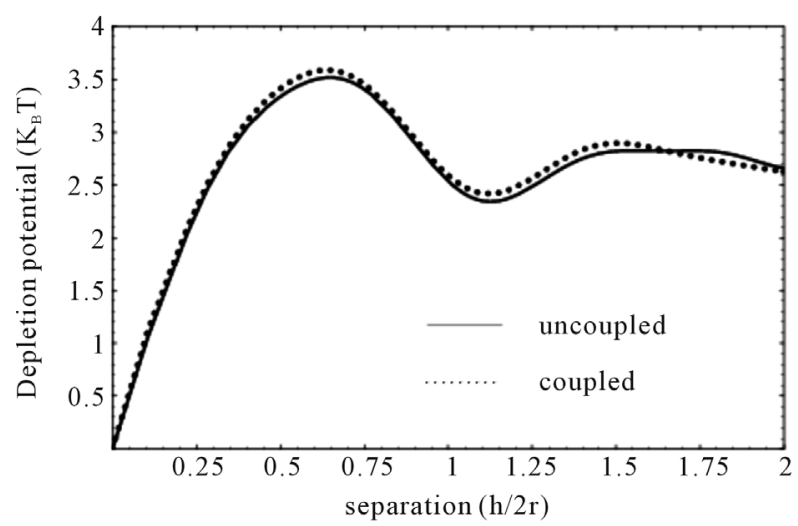

(a)

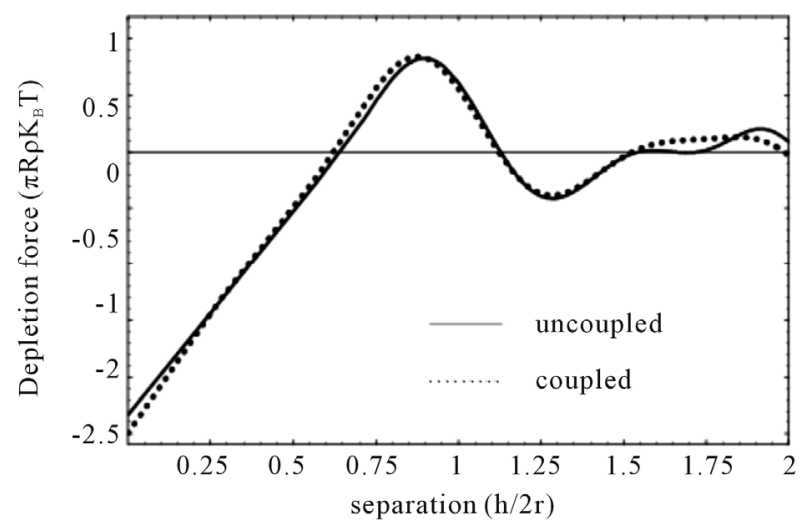

(b)

Figure 4. The depletion potentials (a) and the corresponding depletion forces (b) of the three-sphere system (described by the dashed line) and of the two-sphere system (by the solid line). The volume fraction is $\eta=\mathbf{0 . 3 4 1}$.

-1.45 , so the difference between them is 0.1 ; in Figure 3(b), it is found that the difference of the depletion force of the two systems is 0.2; in Figure 4(b), the difference is also near to 0.2 . It is easy to get that the coupling effect of depletion interactions increase when the volume fraction increases from 0.116 to 0.229 , but it keeps almost invariant when there is a further increase of the volume fraction, such as from 0.229 to 0.314 .

In addition, we also study the depletion interactions in the three-sphere system when the separation of spheres A and $\mathrm{C}$ is changed. So the cases of $H=22 r, 18 r, 16 r$, are studied. For simplicity, only the depletion forces are shown in Figures 5 and 6, which are corresponding to the volume fraction $\eta=0.116,0.229$. In both Figures 5 and 6, the dashed, dotted and solid lines are for $H=22 r$, $18 r, 16 r$, respectively. From Figures 5 and $\mathbf{6}$, it is found that, with decrease of separation, the depletion force of the three-sphere system is increased; compared Figures 5 and $\mathbf{6}$, it is found that the depletion force will increase with the increase of volume fraction. So the coupling effect of the three-sphere system is related to the volume fraction and separation of the system.

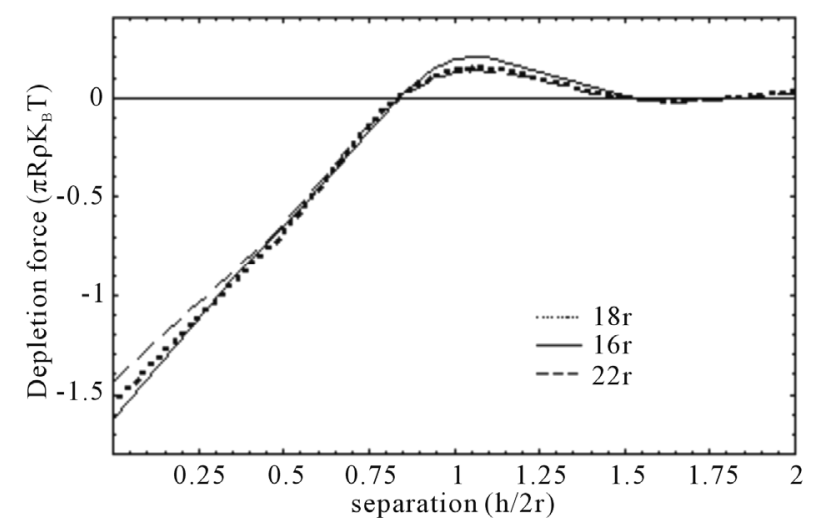

Figure 5. The depletion interaction of the three-sphere systems of $\eta=0.116$ but $H=22 r, 18 r, 16 r$.

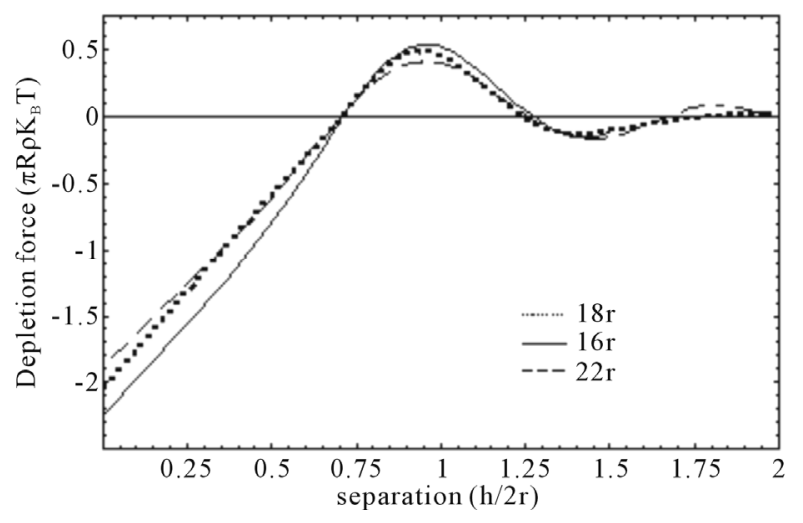

Figure 6. The depletion interaction of the three-sphere systems of $\eta=0.116$ but $H=22 r, 18 r, 16 r$.

\section{Conclusion}

In conclusion, we have investigated the depletion interactions among the three large spheres through Monte Carlo simulations. It is found that the depletion interactions can couple each other and result in a strengthened total depletion forces, and the couple effect will increase when the volume fraction increases from 0.116 to 0.229 ; the couple effect will also increase with decrease of the separation $H$ of the system. In addition, it is also find that, if the third sphere of the three-sphere system is taken as a geometry factor to the other two spheres, the intrinsic or the mechanisms of the effect on depletion interactions from geometry factor and of the coupling effect are the same.

\section{REFERENCES}

[1] S. Asakura and F. J. Oosawa, "On Interaction between Two Bodies Immersed in a Solution of Macromolecules," Journal of Chemical Physics, Vol. 22, No. 7, 1954, pp. 1255-1256. doi:10.1063/1.1740347

[2] B. Götzelmann, R. Evans and S. Dietrich, "Depletion Forces in Fluids," Physical Review E, Vol. 57, No. 6, 1998, pp. 6785-6800. doi:10.1103/PhysRevE.57.6785 
[3] C. M. Xiao and J. Y. Guo, "Depletion Forces in Colloidal System under Geometrical Confinements," Europhysics Letters, Vol. 73, No. 3, 2006, p. 443. doi:10.1209/epl/i2005-10397-2

[4] J. Z. Wu, D. Bratko, H. W. Blanch and J. M. Paausnitz, "Effect of Three-Body Forces on the Phase Behavior of Charged Colloids," Journal of Chemical Physics, Vol. 113, No. 8, 2000, pp. 3360-3365. doi:10.1063/1.1287173

[5] D. Goulding and S. Melchionna, "Accurate Calculation of Three-Body Depletion Interactions," Physical Review E, Vol. 64, No. 1, 2001, Article ID: 011403. doi:10.1103/PhysRevE.64.011403

[6] J. M. Brader, M. Dijkstra and R. Evans, "Inhomogeneous Model Colloid-Polymer Mixtures: Adsorption at a Hard Wall," Physical Review E, Vol. 63, No. 4, 2001, Article ID: 041405. doi:10.1103/PhysRevE.63.041405

[7] D.-M. Zhu, W.-H. Li and H. R. Ma, "On Pair Additivity of the Depletion Force," Journal of Physics: Condensed Matter, Vol. 15, No. 49, 2003, p. 8281. doi:10.1088/0953-8984/15/49/007

[8] W. H. Li and H. R. Ma, "Depletion Potential near Curved Surfaces," Physical Review E, Vol. 66, No. 6, 2002, pp. 61407-61413. doi:10.1088/0953-8984/15/49/007
[9] C. S. Li and C. M. Xiao, "Depletion Interactions in Charged Colloidal System," Acta Physica Sinica, Vol. 56, No. 4, 2007, pp. 2434-2441.

[10] C. M. Xiao, J. Y. Guo and P. Hu, "Geometrical Confinements and Depletion Interactions," Physical Review E, Vol. 73, No. 6, 2006, Article ID: 061403. doi:10.1103/PhysRevE.73.061403

[11] L. X. Wang, H. X. Gao and C. M. Xiao, "Coupling Effects of Depletion Interactions in Colloidal System," Acta Physica Sinica, Vol. 58, No. 8, 2009, pp. 5864-5870.

[12] H. X. Gao and C. M. Xiao, "Depletion Interactions in Cylinder," Physics Letters A, Vol. 362, No. 2-3, 2007, pp. 234-238. doi:10.1016/j.physleta.2006.10.019

[13] C. M. Xiao, J. Y. Guo and P. Hu, "A Monte Carlo Study of Influences on Depletion Force from Another Large Sphere in Colloidal Suspensions," Chinese Physics Letters, Vol. 23, No. 4, 2006, p. 1038. doi: 10.1088/0256-307X/23/4/077

[14] C. H. Bennett, "Efficient Estimation of Free Energy Differences from Monte Carlo Data," Journal of Computational Physics, Vol. 22, No. 2, 1976, pp. 245-268. doi:10.1016/0021-9991(76)90078-4 